\title{
Delivering Multimedia Content in Enabled Cultural Spaces
}

\author{
Christos Sintoris, Dimitrios Raptis, Adrian Stoica, Nikolaos Avouris \\ Human-Computer Interaction Group, Electrical \& Computer Engineering Department \\ University of Patras, GR-26500 Rio Patras, Greece \\ tel. $+30-2610-997349$
}

\{ sintoris, draptis \} @ece.upatras.gr, \{stoica, avouris\}@upatras.gr

\begin{abstract}
In the context of a project involving development of an electronic guide and an educational application for a historical cultural museum, we defined an architecture that permits efficient and effective co-existence of objects in the physical space and digital multimedia content. Handheld devices were used for mediating the process. The prime consideration of the developed architecture was the optimal delivery of multimedia content, mainly in the form of audio but also video and text, to users of handheld devices, which raises an interesting number of issues both in terms of technology used and human-computer interaction.
\end{abstract}

\section{Categories and Subject Descriptors}

\section{C5.3 [Computer System Implementation]:}

Microcomputers - Portable devices.

H5.1. [Information Interfaces and Presentation]:

Multimedia Information Systems- artificial, augmented and virtual realities.

H5.2. [Information Interfaces and Presentation]: User

Interfaces - user-centered design, interaction styles.

\section{General Terms}

Design, Experimentation, Human Factors

\section{Keywords}

Mobile devices, multimedia, context, personalization, enabled space, usability, interaction

\section{INTRODUCTION}

Handheld devices, such as cellular phones and PDAs, have greatly evolved over the past years and this trend shows no signs of slowing down. Handhelds are evolving towards bundling more multimedia capabilities (image capturing and storing, music, TV etc), services (e.g. navigation assistance) and connectivity options (e.g. HSDPA) under the same hood. This continuous technological development takes place while we observe an increased use of these devices in everyday life by millions of people around the globe.

Permission to make digital or hard copies of all or part of this work for personal or classroom use is granted without fee provided that copies are not made or distributed for profit or commercial advantage and that copies bear this notice and the full citation on the first page. To copy otherwise, or republish, to post on servers or to redistribute to lists, requires prior specific permission and/or a fee.

MOBIMEDIA 2007, August 27-29, Nafpaktos, Greece

Copyright $\odot 2007$ ICST 978-963-06-2670-5

DOI 10.4108/ICST.MOBIMEDIA2007.1938
Given the new advanced technological capabilities and the widespread use of these devices, there is a transition of the typical use from mobile communication devices to user companions, servicing communication, entertainment and personal needs of their owners. However nowadays, despite their high capacity, handheld devices provide mainly connectivity and content to their users without sensing the social and physical context $[3,9]$ in which they exist and thus are not able to adapt their services to the specific conditions and needs of their user. Therefore the device must become a tool that can interact with the surrounding social and physical space, access services and information according to the user's profile and needs.

\section{ENABLED SPACES}

By the term enabled space we characterize a physical space that has the ability to interact with mobile or ubiquitous devices and to serve its visitors in completing their tasks. An enabled space must have the ability to take advantage of certain technologies and provide to its "inhabitants" new services and possibilities. An example of an enabled space can be a library. In a typical library, a visitor can browse a catalog in order to locate a book by using a desktop PC. Then he must find the room and the shelf that this book is located. An enabled library can reformulate the task of locating a book by providing new services such as: the ability to sense the visitor's position and give him directions on how to find a shelf, to prompt and inform him that there is a book near him that fits his profile and interests, to allow him to get extended information for a book.

From our point of view, an enabled space must be able to allow a user to perform all the typical activities that are already possible inside this space in an easy and effective way, while providing new specially-designed services (or extending existing ones) by exploiting the technological characteristics and providing multi-facet content according to user position and needs.

There are various prerequisites that need to be satisfied in an enabled space. The most significant one is related to the services that one chooses to provide to the users. In order to design these services the first step is related to understanding the motives and the actions that the users perform inside this space and then to study how the technological decisions will influence the typical users. The technological decisions must address issues like: a) how the physical space needs to be altered in order to become enabled (e.g. is there a need to install a wireless network infrastructure in order to sense the visitor's location?), b) how these technological decisions will influence the interaction between the user and the space and also how the interaction will be influenced by the services that are provided, c) the type of devices that the user will use inside the space (e.g his personal cellphone or a special purpose device, a mobile device with a screen or just a headset), d) the cost of the 
approach. In a typical scenario an enabled space must provide communication capabilities to the visitors (for example by using WiFi access points or Bluetooth transmitters), sense the location of the visitors (either in an indirect way, e.g. by knowing the visitors' last position from a recent scan of an RFID tag, or by sensing that he entered a room because he established a link with a infrared beamer, or in a direct way, e.g. by using indoor location tracking technology such as indoor GPS, UWB positioning [10], WiFi positioning or Cricket positioning [6]) and provide new services that take advantage of the coexistence of virtual and physical space .

Concluding, the selection of the most suitable technology in order to produce an enabled space is mainly driven by the actions and the tasks that visitors can perform inside this space, by the applications that are going to be deployed inside the space and by the capabilities of the targeted mobile devices.

\section{MUSEUMS AS ENABLED SPACES}

Museums can become typical examples of enabled spaces. They can be described as cultural information spaces, where a visitor may interact with the surrounding physical space by observing and studying the exhibits, either alone or as a member of a group (in most cases). The vast majority of visitors inside a museum have a specific motive: to learn more about the exhibits by studying them as physical objects, by having access to additional information about them, (artistic, historical, cultural, social), or by correlating the exhibits with each other or with well known facts and by seeking answers to new questions that have been produced during this learning process.

In order to support the motives and the needs of the visitors, various applications and systems have been developed during the last years aiming at creating a new virtual dimension inside the museums. Sotto Voce [11] is a project which promotes research in physical, computational, and social sciences. In one of its aspects it involves an electronic guidebook designed to facilitate interaction between companions and to help visitors engage with a museum environment. It has been developed and evaluated at Xerox PARC and deployed at Filoli, a historic house in Woodside, California.

Exploratorium [5] is a public science museum in San Francisco where visitors have direct contact with the exhibits by touching and manipulating them and, as a result, receiving information about various scientific phenomena that occur in front of them. It aims at providing personalized access to the visitors by delivering mobile web content and by giving them the opportunity to create their personal web page with notes, favorite exhibits, results, either before or after their visit. The visitors can extend their interaction with the exhibits by using a networked handheld device to use for taking measurements, data collection, and other experimentation and thus better understand the phenomena demonstrated by the exhibits.

\subsection{The Case of Solomos Museum}

During the last years we have been engaged with designing and developing a full fledged museum system, including digital data and media repository, a website and services suitable for mobile users, for a cultural and historical museum [1], the Solomos Museum located at the island of Zakynthos, Greece.

A core idea behind this project has been, besides rendering the main halls of this Museum "enabled" and thus improving the visitor's experience, to support the visitor before and after the visit. The visitor can browse through the exhibits in the museum website and create in advance his own personal trail that he would wish to follow inside the museum. In addition the visitor may re-live his experience after the visit by looking back to the exhibits that he found to be interesting.
To support the above scenario technological decisions were made that in effect influence the application architecture. After extensive testing and development of mock-up applications it was decided to drop initial support for a Java based solution and to use Microsoft's .NET Compact Framework as a platform for developing the handheld applications, since it provides, in our view, a developing platform better suited for our goals. The final solution runs on the Pocket PC operating system with the aim to support in the future also the Smartphone platform.

In terms of positioning technologies, we evaluated a number of solutions including WiFi, Ultra Wide Band, ultrasound and RF, infrared beacons and Bluetooth. We decided to use RFID technology as a way for indirectly determining position information, a decision which was taken on the basis of cost effectiveness and minimal intrusion in the museum's infrastructure.

Content delivery is initiated by the visitor through actively scanning an RFID tag using the handheld device, and performed through a WiFi infrastructure. The digital content resides in separate servers, servicing a) audio, b) textual descriptions, images and video, c) educational applications and other applications (as notes exchange). Effort was made to choose the relevant technologies based on extensibility and effectiveness relative to cost and on using well known and widely used technologies, as discussed in section 3.4 of the paper. Textual descriptions, along with images and text are delivered to an embedded web browser using a standard http server and thus contain hyperlinks, as another form of initiating content delivery, by referring to other exhibits or to additional information for an exhibit. Control messages are exchanged between the devices and the servers by the XMLRPC protocol.

\subsection{Content Structure}

Having in mind that the visitor has a natural presence inside the museum, there were various issues that needed to be addressed regarding the digital content that is going to be presented to the users. Before outlining and discussing those, the organization of the digital repository is going to be briefly presented.

The digital content of the Museum resides in two clearly separated databases. In the frame of the project, the Museum has digitized it's collection, creating an extensive repository based on the ICOM $\backslash$ CIDOC Conceptual Reference Model [2] for Museum metadata. For the purposes of the PDA assisted visit, a second, multimedia database hosts the necessary multimedia content, along with spatial information concerning the layout of the museum's halls and the positions of the exhibits. Explaining the the databases structure in more detail, is beyond the scope of this paper and the two databases are treated from now on as one digital repository.

Regarding the form of the content, the repository holds information as: Textual descriptions, audio narrations, images and video.

In a conceptual level, the content is organized along a set of properties. Content might be:

- limited to describing and presenting an exhibit,

- referring to prevailing conditions of a realm (such as social or economical) related to an exhibit,

- providing biographical or other information about a relevant personality,

- laying out secondary properties of an exhibit, such as the materials it consists of, the techniques used by it's creators etc.

- material that would otherwise be not accessible to the visitor due to the physical layout or exhibit characteristics (a detail of a painting that is not easily visible, pages of a very old book that could not be 
otherwise touched, the sound of a musical instrument etc).

The above multidimensional organization of the content is invoked by the use of the CIDOC framework for organizing the digital repository.

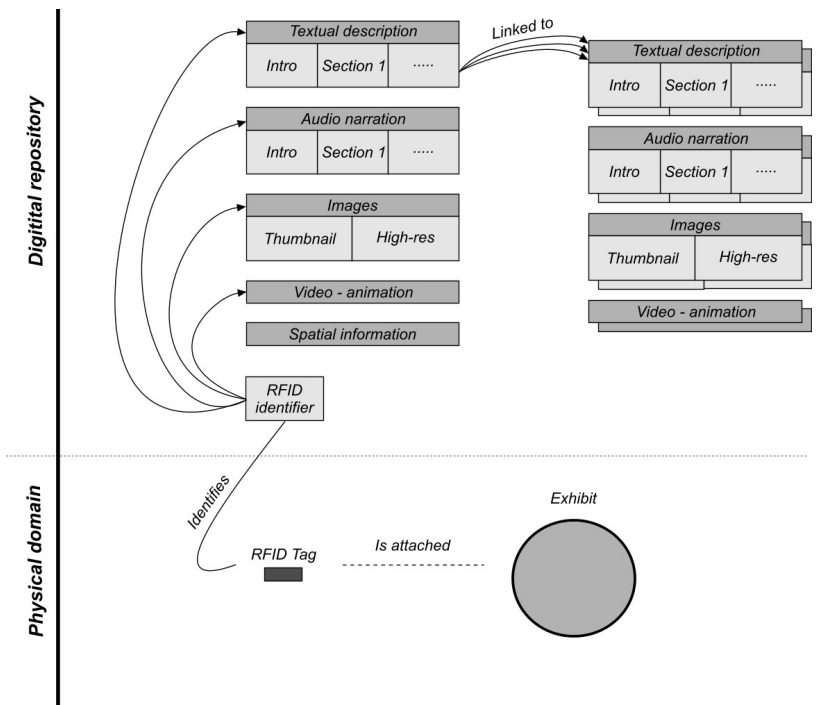

Figure 1: Conceptual layout of the digital repository

The mapping between the physical exhibits and the digital repository is based on the RFID tags. The exhibits are assigned each an RFID tag (with the exception of the smaller exhibits, which are organized in clusters and each such cluster is identified by one RFID tag). To each and every RFID identifier in the repository, content is assigned, in the form of textual description and an image of the exhibit. Audio narrations and videos are not available for all exhibits. Most textual descriptions are organized in sections. The PDA, after scanning an RFID tag, initially displays the introductory section. The consecutive sections are displayed only if the visitor explicitly requests it and contain more detailed information. Hyperlinks in the text refer to other textual content.

During the actual visit these types of content do not have the same importance. Having in mind that the visitor actually sees the exhibits and that we do not need to force him to constantly look at a small PDA's screen, the role of the multimedia content for the users of the handheld devices is complementary and it enriches and augments the physical space of the museum. Therefore, the main method of delivering content is through audio narrations and the main role of images is for confirmation that the visitor is reading the correct description or listening to the correct narration.

Besides the RFID tags which are explicitly assigned to single exhibits or clusters of exhibits, the digital repository also contains content that refers to RFID tags that identify an specific hall or a thematic group of exhibits. This content serves as an introductory guide to the specific part of the museum.

Finally, a considerable amount of the digital repository's content has no direct reference to physical exhibits or RFID tags. This concerns additional information (e.g. a biographical note or a description of the prevailing social conditions of a historical period) and is linked on a secondary level with the exhibits through hyperlinks contained in an exhibit's textual description.

The bulk of the information which is residing in the digital repository is in the form of textual descriptions, which are interconnected through hyperlinks and contain additional media such as images and video.
For the most important exhibits the repository contains audio narrations. This audio content is narrated in sections, similarly to textual content. If applicable, sound bites (such as the sound of an old piano) or video sequences are also included. (Figure 1). We must note that in order not to isolate the visitors (in most cases people are visiting a museum in groups) the audio is delivered not only through one-ear headsets but also through the device's speakers, allowing the visitors to select the preferred method.

In short, the system provides:

- multimedia content attached to specific exhibits,

- multimedia content linked to clusters of exhibits,

- multimedia content of introductory and general nature in the cases where a user starts using the system, starts a predefined route, enters a room or observers a cluster of exhibits.

- content related to an exhibit or group of exhibits such as the historical or social context or an important person.

The organization of the digital content follows only loosely the physical hierarchy and the layout of the exhibits in the museum, starting from the museum as the top entity, through exhibition rooms, to showcases, reaching finally the level of single exhibits.

The hybrid character of the enabled museum space, containing both physical objects and related digital information, results in mappings between two spaces with different content density. I.e., there are physical objects with high density of digital content associated to them, while on the other hand, whole clusters of objects have just rudimentary associated information. In other words, the amount of digital content does not reflect the physical presence of the exhibits. This is reflected in the navigation patterns of the users of the enabled space, who may spend considerable time either navigating the physical or the digital space.

\subsection{APPLICATIONS}

In order to support the visitors' motives, which we presume is the need to learn and be entertained, our system comes in two manifestations:

- Mobile guide, aiming at guiding the visitor optimally through the museum.

- Stimulating educational activities, for groups of young visitors, e.g. school parties.

The rationale behind this choice is that the system should be able to support the needs of both adult visitors (the case of the mobile guide assistant) and the needs of school parties or children of up to the age of fifteen (the case of the educational activities).

\subsubsection{Mobile guide}

In both cases the applications use in a large extend the same infrastructure. The digital repository, the content delivery and other crucial parts of the system are shared by both applications. What changes is the implementation of the applications on the mobile device as well as the use of an application server servicing the educational activities. The interaction with the museum's physical space is facilitated by two concurrent modes:

- On one hand the visitor walks around the museum and collects information by observing the exhibits and their arrangement in the museum space. At the same time the visitor can scan the exhibits' or the rooms' RFID tags and collect additional information 
from the digital domain. (The physical and the digital domain thus spatially overlap).

- On the other hand the visitor can use the handheld device to follow hyperlinks and eventually get information which is not directly related to the exhibits within his immediate vicinity or, in some cases, not directly related to the museum at all.

There is a strong relation between the digital content and the physical space, so there is a need to parametrize system behavior according to the context in which the actions of the users take place. The following scenario demonstrates this need: A visitor scans the RFID tag of an exhibit - e.g. the uniform of a historic person - and as a result the handheld device starts reproducing an audio narration, displays a small text and shows a thumbnail image of the exhibit, for the purpose of visual identification of the related exhibit. The system can infer by the RFID scan that the visitor is actually standing in front of the exhibit. By clicking on a hyperlink on the PDA, the visitor sees information of a related item - a portrait of that same historic person - which is however not located in the immediate vicinity, only this time the image is more detailed, since the visitor has no physical access to the second item and thus more visual information is considered to be useful.

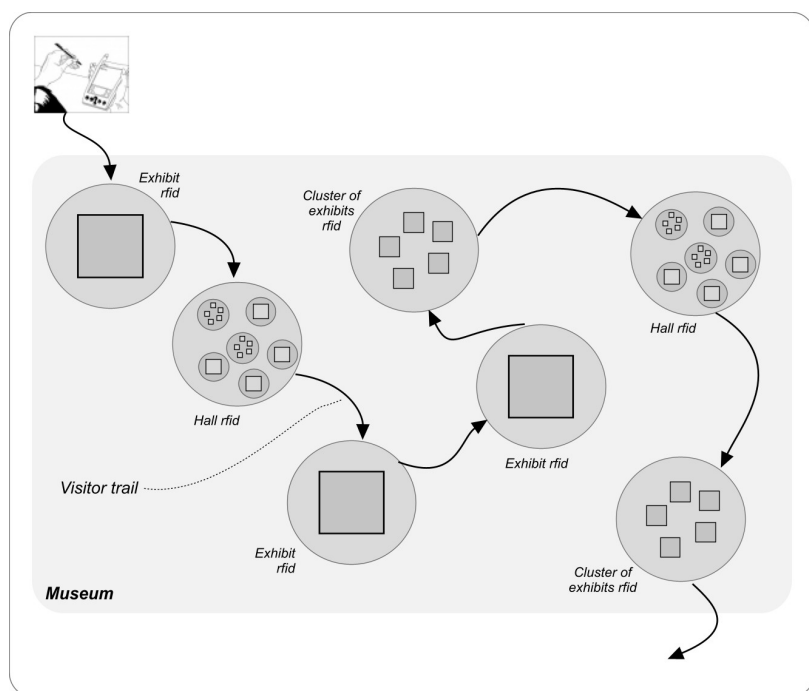

Figure 2: Interacting with the enabled museum

Considering the ways in which a visitor can have access to content, we can distinguish the following scenarios (Figure 2), influencing the content delivery process:

1. The visitor can scan an RFID tag with his handheld device. This results in: (a) gaining information about his exact position in the museum and (b) in delivering to his device a textual description accompanied by a thumbnail of the exhibit (servicing visual verification that the correct description is at hand) as well as synchronized audio and video (if applicable).

2. The visitor can point to a hyperlink in the textual description and can receive further digital content about the same exhibit.

3. The visitor can point to a hyperlink and receive content about an exhibit not in his immediate vicinity. In this case, the thumbnail image will be replaced by higher resolution images.
4. Finally, the digital content received might be referring to an object, person or concept which is relevant to an exhibit but does not strictly reside inside the museum.

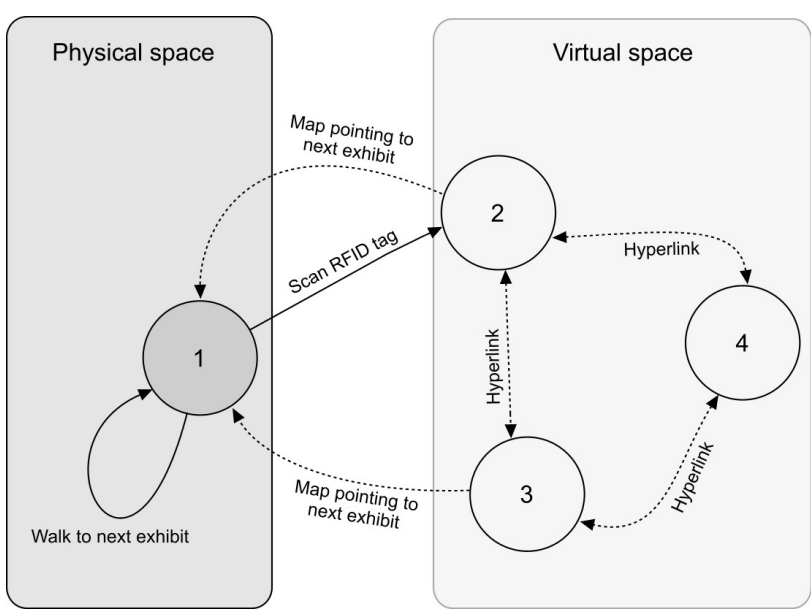

Figure 3: Access to content depending on physical proximity and relevance

In addition to the above, the type of visit may also influence the content delivery process. The mobile guide application allows the visitors to select among various types of predefined routes (time related, topic related, or a personally defined route, designed beforehand using the museum website) or to freely interact with the exhibits. In that context, time influences for example the extend of the content delivered. In the case of the free visit the behavior of the system is limited to responding to the requests of the user when interacting with different exhibits. On the other hand, when the user selects a predefined route the system must guide him in order to find the next exhibit of the route. This guidance happens in two ways: the interface provides a link to the next exhibit (when the user selects it, the system assumes that he is in front of the exhibit and starts the narrations) and provides the location of the next exhibit on an interactive map and waits for the user to scan the RFID tag in order to start providing him with information. When a user accidentally or by choice selects an exhibit that does not belong to the route the system flexibly allows him to get information about it but points him, through a hyperlink and the interactive map, to the next exhibit in the predefined route.

Finally, content is shaped by the importance that the museum curators assign to a specific exhibit. Important exhibits, representative of the museum's collection, are accompanied by a plethora of multimedia material, while less important items of the collection, are organized as clusters of exhibits, are mentioned with less detail (e.g. individual coins in show cases).

\subsubsection{Educational activities}

The main goal of educational activities, which are also supported by the mobile devices, is to make the museum's accumulated knowledge more attractive and accessible to young visitors. The educational activities are built on top of the infrastructure for the mobile guide sharing with it, among other, the rendering and streaming functionalities. Some of these activities involve collaboration of visitors while others are suitable for individual use. The core idea behind these activities is to engage pupils in a stimulating game play, that allows them to assimilate knowledge in a seamless way. The games involve virtual objects (text and sound extracts as well as images) which are correlated to exhibits. To progress from one game level to another, the students must use both physical and virtual elements and need to make practical use of the information 
available to properly associate digital elements with physical objects or with other digital elements, exchange digital objects between them etc.

An example of such activity is presented in [8]. Figure 4 shows a team of children using the PDA during playing an educational game in the Museum.

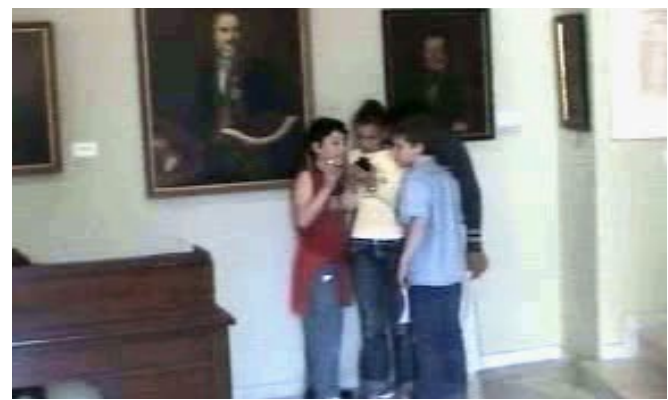

Figure 4. A team of children using the PDA

\subsubsection{Visit preparation and post-visit support}

The system was designed to support both visitors in the museum, as well as pre-visit and post-visit activities.

Both the mobile guide and educational activities support visit preparation and visit follow up. Using the museum web site, visitors can prepare their visit by choosing among various preferences, such as types of visits etc. When the visitor enters the museum his profile and preferences are transferred on the handheld device to allow him a personalized visit. Afterwards, the visitor can get back on the website and recreate and reproduce his visit by reviewing the exhibits he asked information about, or to which he attached new media and comments, through his personal account. Especially in the case of the educational activities, an educator can register in the museum website and prepare the visit of a class by creating the desired visit scenario. After the visit he can review with his students in the classroom the activities and the trails of the individual students.

In addition to the review of one's trail, the user can use the PDA in order to produce his own personal content by marking favorite exhibits or by attaching various annotations and comments to an exhibit, which can then be available to other users, provided that the visitor allows this. Examples of such media items are text comments, drawings and voice messages. In addition, the visitors can browse through the media items of other visitors or review their experience later on by browsing through their comments on the museum's website.

\subsection{Integration and Scalability}

In order to render a physical space enabled and to integrate such mobile services, it is necessary to tackle various issues and to adapt to the characteristics of a physical setting. Special effort has been made to design a solution that can be both easily adapted to new environments and extended to support a broader user base and services. Nevertheless, the steps needed to integrate such a solution to an existing setting are not trivial:

- The existing legacy information system has to be wrapped, extended and equipped with proper interfaces in order to be integrated with the mobile guide application.

- The multimedia content of the museum must be adapted in order to be presented in the handheld devices (transformed to lower resolution and adequate image sizes), while new digital content (audio narrations) has to be produced.

- The museum's infrastructure has to be enhanced and "enabled" by relevant technologies in the form of access points, RFID tags, public information screens etc.

- The mobile services which handle the information and multimedia content delivery have to be adapted.

- Integration of all the components in order to create a single system respecting the museum character and mission.

The current system needs to serve only 10 client devices thus being not very demanding and allowing some architectural components to be simplified. This does not mean that the design does not provide for a possibly significant growth of the number of client devices. The system aims to be easily extended in various directions both in terms of features and in terms of hardware configuration. Also it can scale up with just a few modifications at the operational level. Extending the features of the system can be realized for example by adding new modules implementing educational activities. The development of these modules must be based on a set of interfaces that allow consistent handling through code reflection technology.

From another perspective we can look at extending the system hardware configuration by adding more devices or even servers. The mobile guide application has a zero configuration service for the mobile devices that allows fast and easy operations like adding new devices or initializing devices for various visitors. In our case some of the server applications (digital content, audio narration streaming, application servers) are collocated on the same physical machine but the design allows specialized servers for each of them or even distribution of a single server among multiple machines.

This "extensible design - simple implementation" approach is visible in the current implementation of the streaming server. A standard HTTP server has been used for delivering media files to the client devices. This choice is considered sufficient for the current needs and in particular for the reduced number of concurrent clients. To accommodate in the future more client devices the simple HTTP based streaming media server could be transparently replaced by a specialized and more efficient one. The only modification needed is just to replace the server side component. The client implementation is streaming-ready.

In addition the narrations are produced by recording human readers of the various texts. A desired extension of the system is to add a module that produces the narrations on the fly using speech synthesis. This approach is not only cost effective but also enables the updates made in the texts to be propagated immediately to the narrations.

The presented architecture of the system and the model to deliver multimedia content to mobile devices can thus be easily applied to other spaces. The novelty here is the integration of multiple media resources to complement and support the current state of the user, as discussed above.

\section{FUTURE WORK}

This project has reached a prototype status and the ongoing work offers many possibilities for expanding its capabilities into various research areas. On the technological domain we are focusing on how to integrate various mobile devices with different operating systems. Our aim is in the future, each visitor to be able to use the museum's application on his personal mobile device (i.e. his cell phone), instead of the museum's PDA.

Currently, the interaction between a visitor and an exhibit is based on the scanner metaphor, since the visitor must scan the RFID tags in order to have access to multimedia and information. Other metaphors are the autopilot metaphor, where the system responds autonomously, depending on the visitor's 
location and the remote control metaphor, where the visitor uses the mobile device in a way similar to the use of a television remote control. We are specially interested in experimenting with the autopilot metaphor by attaching accelerometers to mobile devices. This will allow a visitor to just point-and-click to the exhibit of interest. In addition, besides knowing the exact physical object a visitor is pointing at, such an approach will yield also information about the visitor's exact position at any time.

Through various types of usability evaluations regarding the system (concerning the interaction metaphors, the characteristics of the mobile devices, the effect of natural conditions such as light and noise, etc.) we are aiming in producing abstract guidelines that will be general enough in order to contribute to the design and the evaluation procedure of producing context aware media rich mobile applications.

Another direction of research is to investigate the value that mobsites [7] could bring into the museum. Mobsites are mini web servers running on mobile devices. Through them, the museum will have a synchronous on-line media source in a state of constant change, in relation to the visitors' live movement, their current activities and their distribution in the physical space.

Also not of minor importance is the aim of creating a framework to ensure security and privacy of the users' data. The ability of devices to be connected to various networks, to be used as tracking devices for the users' movement and actions, as well as the multimedia capabilities to record sound and video makes them equally useful and vulnerable from the point of view of the security of personal data.

Finally, our main target is to expand the results that are produced while designing this museum application, to other enabled spaces in a concrete and unified way. We plan to design and develop an infrastructure that will be able to facilitate various mobile services suitable for different enabled spaces in the form of plugable mobile applications, to provide the ability to the users to interact with different spaces by using their own mobile device through one consistent interface, and to provide advanced personalized access to these spaces by adopting user modeling techniques based on previous interactions with other spaces and the users' preferences.

\section{CONCLUSIONS}

The Solomos Museum project so far shows that a clear benefit can be obtained from inserting mobile content delivery technology in a cultural information space. We have identified a set of requirements with regard to the functionality of such a platform. Also, tracking the movement and behavior of persons, even with their consent, raises a number of serious privacy issues which are governed by strict laws, an area of particular concern that requires additional effort.

On a different level, considerations with regard to the scalability of our solution to allow larger museums to use such a platform also need to be explored in more detail. Ways to transform the system to be able to support different types of information spaces need also to be further investigated.
In addition to documenting our experiences with a real-life mobile content delivery system, this paper raises a number of questions on why and how multimedia content can effectively and efficiently be used to benefit mobile users, regarding the design and development of the proper infrastructure, the enabling of spaces, the selection of proper content and methods to deliver it and the design and development of usable and accessible mobile systems. These are crucial issues that need to be addressed in order to advance the development of mobile services for multimedia content delivery.

\section{ACKNOWLEDGMENTS}

Partners of the Solomos Museum project are the University of Patras, Globo Technologies SA, Ionian University. Iblind and SingularLogic. The research presented here is partially supported by the Greek Secretariat for Research and Technology (GSRT) Program PENED2003, Grant 03ED791 Hybrid Libraries Project.

\section{REFERENCES}

[1] Cabrera, J. S., Frutos, H. M., Stoica, A., Avouris, N., Dimitriadis, Y., et al. Mystery in the Museum: Collaborative Learning Activities using Handheld Devices. Proc MobileHCI 2005, Salzburg, Austria, Sep. 2005, pp. 315-318.

[2] ICOM/CIDOC Documentation Standards Group (2004), Definition of the CIDOC Conceptual Reference Model, version 4.0, 2004, available from http://cidoc.ics.forth.gr

[3] Dey, A. Understanding and Using Context. Personal and Ubiquitous Computing. Journal, Vol. 5(1), 2001, pp.4-7.

[4] Dourish, P. What We Talk About When We Talk About Context. Personal Ubiquitous Comput., Springer-Verlag, Vol. 8, No. 1., February 2004, pp. 19-30, 2004.

[5] Exploratorium. Electronic Guidebook forum Report, San Francisco, 2005. http:// www.exploratorium.edu

[6] Nissanka, B. P. The Cricket Indoor Location System. PhD Thesis, Massachusetts Institute of Technology, June 2005.

[7] Nokia Research Center, Mobile Web Server, available at http://research.nokia.com:

[8] Papadimitriou I., Komis V., Tselios N., Avouris N., Designing PDA Mediated Educational Activities for a Museum Visit, Proc. of CELDA 2006, Barcelona.

[9] Raptis, D., Tselios, N. and Avouris, N. Context-based design of mobile applications for museums: A Survey of existing practices. Proc MobileHCI 2005, Salzburg, Austria, Sep. 2005, pp. 153-160.

[10] Ubisense. available at: http://www.ubisense.net/

[11] Woodruff, A., Aoki, P., Hurst, A. and Szymanski, M. Electronic Guidebooks and Visitor Attention. Proc. Cultural Heritage Informatics 2001, Milan, Italy, 2001, 437-454. 